\title{
Quantifying Dominance in the MULTISIMO Corpus
}

\author{
Maria Koutsombogera, Rachel Costello and Carl Vogel \\ Trinity Centre for Computing and Language Studies \\ Computational Linguistics Group \\ School of Computer Science and Statistics \\ Trinity College Dublin \\ The University of Dublin \\ Dublin 2, Ireland \\ $\{$ koutsomm, costelra,vogel $\} @$ tcd.ie
}

\begin{abstract}
This paper is concerned with investigating conversational (verbal and non verbal) features related to conversational dominance and discovering evident relations between these features, personality traits and perceived dominance scores. Ordinal regression models were applied to find associations between dominance scores, both as a predicted value and as a predictor, and personality traits and verbal features. Results report the association of high dominance scores to the large number of words a speaker utters per minute, as well as to high extraversion and high openness traits. Low dominance scores have been associated with high agreeableness.
\end{abstract}

\section{INTRODUCTION}

Conversational dominance can be viewed as a dynamic state in a dialogue, which a dialogue participant manifests through verbal and non-verbal activity in an attempt to control the conversational actions of his/her co-participants [1], [2], [3], [4]. Multiparty conversations often feature a dominancesubmission construct, where one person typically adopts a central role, thereby dominating both the conversation and its result. Dominance has been largely studied in social psychology due to the general consensus that it is possible to infer the dominant character of a conversation using a variety of communicative verbal and nonverbal cues [5], without any prior knowledge of that person's background information [6].

\section{A. Prior research}

Research on automatically estimating conversational dominance has established a number of quantifiable dimensions among which the most significant are related to the verbal channel and turn-taking, such as speaking length, number of words and overlapping speech. Speaking length and number of words have proved to be especially robust in the prediction of dominance [7], [2], [5], and it has been suggested that they may be the most important determinants in expressing and perceiving dominance [7]. The interlocutor who articulates the greater number of words, and potentially speaking for a greater length of time, can be described as controlling the other speaker.

Gaze information has also been viewed as closely related to conversational dominance, co-examined with the aforementioned linguistic features. Kendon [8] reported on observable gaze direction patterns systematically related to features of talk and important to the regulation of turn-taking in conversation. The hypothesis previously reported is that conversationally dominant people receive more frequent and longer-lasting gazes from the other interlocutors [5], [6]. Gaze features such as the duration of gazing at others, the amount of received gaze and the amount of mutual gaze among dialogue participants have been suggested to be strong indicators for predicting dominance.

Several studies related to dominance and leadership estimation employ human judgements of dominance levels of dialogue participants, in the form of either self- or otherassessments [9], [2], [5], [4], [6]. These judgements in turn serve as the ground truth to which linguistic or behavioral features are associated. An iterative question that arises, is who would best qualified to act as a judge, i.e. participants themselves or external observers. Participants are more likely to have a biased approach, giving their co-interlocutors a more favourable rating[10], and may be incapable of accurately seeing their own behaviour, as they are too invested in the reasoning for their actions [4]. Thus, observers seem more objective and more likely to make assertions based on dispositional attributions. For this reason, we have employed external annotators for this study.

Conversational dominance has received a lot of attention as it is considered, apart from a mechanism related to turn organisation, an aspect of personality. Attention has been given to drawing relationships between personality traits and leadership style. Furthermore, strong correlation has been found between social dominance and leadership [11], with dominance being one of the key characteristics of specific styles of leadership [12], [13]. However, there is little work investigating the direct association of dominance to the BIG5 personality framework ${ }^{1}$ in terms of conducted empirical study [14].

\section{B. A new data source}

For this work we have used 18 dialogues from the MULTISIMO corpus [15], a multimodal corpus originally consisting of 23 sessions of collaborative group interactions, where two players work together to play a quiz and are guided by a facilitator, who monitors their progress and provides feedback when needed. The task of the players was to discuss with each other, provide the 3 most popular answers to each of 3 questions, and rank their answers from the most to the least popular. The corpus' overall duration is approximately 4 hours, with an average session duration of 10 minutes. This dataset

\footnotetext{
${ }^{1}$ The five personality traits are: Extroversion, Agreeableness, Conscientiousness, Neuroticism and Openness to Experience.
} 
addresses multiparty collaborative interactions and aims at providing tools for measuring collaboration and task success based on the integration of the related multimodal information. In this study we exploit the linguistic features, personality scores and dominance scores of the 2 players in each group, as well as gaze exchange patterns among all 3 participants in each group.

\section{Hypotheses}

Our main research question is to determine the relationship between the portrayal of conversational dominance, as assessed by observers, and both BIG5 personality traits and conversational features.

Particular emphasis will be placed on determining if there is positive correlation between extroversion and the manifestation of dominance, for the reason that extroversion levels are the core influence of a person's tendency to engage and attract social attention [16]. Studies conducted explicitly on extroversion maintain agreement that a high score on this trait corresponds to a greater likelihood of exhibiting dominance [17], [12]. Agreeableness has been associated with dependent and submissive behaviour [17], so it would subsequently be expected for dominant people to have a low level of agreeableness. Constructs relating to dominance have been associated with a negative correlation for agreeableness [18]. Conscientiousness has loosely been supposed to not have any impact of the indication of dominance whatsoever [17]. A similar situation occurs with neuroticism, as neither specific nor conclusive enough evidence exists for its relation to dominance.

We also hypothesize that large volumes of linguistic features indicating ownership of the floor, i.e. the number of words and number of words per minute that a speaker utters, as well as a speaker's turn duration, may correlate with high dominance scores.

Furthermore, gaze was viewed as an extremely important and informative feature [4], [19], [5]. Thus, we perform a partial analysis to test the hypothesis that the gaze information a facilitator provides carries weight in determining the most dominant participant.

The remainder of this paper is organised as follows: Section $\S$ II presents the methodology followed and the features taken into account. The following sections describe the experiments with dominance as a predicted value ( $\S$ III) and as a predictor ( IV) and we conclude with a discussion of the results $(\S \mathrm{V})$.

\section{METHODS}

\section{A. Conversational Features Extraction}

The dialogue audio files were segmented in speaking turns and were manually transcribed. ${ }^{2}$ Transcription includes the speakers' identification and the words they utter, as well as the marking of silence and overlapping talk. Linguistic features were then extracted form the annotated data. Although initially a large set of turn-taking and verbal features was derived, a smaller set was chosen for this study; for each participant, the following audio features were computed:

\footnotetext{
${ }^{2}$ Transcriber (http://trans.sourceforge.net/) last accessed 10.05.2018.
}

- Number of Words: The total number of words uttered by a participant during the conversation.

- Number of Words per Minute: The number of words uttered by a participant on average over the course of one minute of his/her speaking time.

- Total Number of Turns: The cumulation of the number of turns a participant takes over the course of the session.

- Average Turn Duration: The speaking time per participant divided by the participant's number of turns to discover the average length of turns in the session.

\section{B. Personality Traits}

An aim of the corpus collection has been to investigate the effect of personality traits on the task success but also on participants' collaborative behavior. Personality variables are an important tool for the interpretation of social behavior. Also, the related literature widely acknowledges the necessity to have an accepted classification scheme to categorise empirical findings and that the 5-factor model is a robust and meaningful framework enabling the formulation and testing of hypotheses related to individual differences in personality [20]. We therefore opted for the BIG5 personality measures that assess the following five traits: Extroversion, Agreeableness, Conscientiousness, Neuroticism and Openness to Experience. Before the recordings, participants completed the Big Five Inventory (BFI), a self-report inventory designed to measure the Big Five dimensions [21], [22]. The test consists of 44 items (statements) and the participants rated each statement to indicate the extent to which they agree or disagree with it. As a result, a list of scores per personality trait and per participant was created. The percentile rank of each participant across the five personality traits was then calculated using local norms, i.e. the norms were constructed upon the groups population.

\section{Dominance Assessment}

A perception experiment was run to collect ratings from a group of annotators who observed the dialogue videos and assessed the level of dominance of the involved players. These ratings serve as the ground truth to which the set of extracted features would be compared. Five annotators were asked to watch the set of videos and assess the dominance levels of the 2 players in each video. The annotators were asked to watch each video twice and rate the 2 quiz players on a scale from 5 (very dominant) to 1 (not dominant at all), depending on how dominant they think that the video speakers are. Annotators completed the ratings after watching the full videos and they did not spend more than 1 hour per day on the task. Raters were also supplied with the explicit definition of dominance as "a person's tendency to control the behaviour of others when interacting with them" and were informed of the general assumption that dominant individuals are more active in terms of multimodal cues, i.e., that they both move and talk more and do so more often than non-dominant people. They were not briefed however, on what specific verbal or nonverbal cues to scan for, so the judgements would not be biased.

In order to create one individual dominance score for each participant, rather than a set of scores, the median dominance 
score was determined from each participant's respective set and assigned to that participant as their conclusive conversational dominance score. The Intraclass Correlation Coefficient (ICC) was used to measure the reliability of the given ratings, i.e. to determine the level of agreement among the annotators' decisions on their dominance ratings for each individual. The ICC was established to be 0.776 , indicating a good level of agreement among the raters $\left(p=3.57 * 10^{-6}, 95 \%\right.$ confidence interval for ICC population values: $0.559<$ ICC $<0.886$ ).

\section{Gaze Features}

Gaze has been considered an important feature for dominance prediction. To study the gaze behavior of the participants in the corpus, gaze shifts were annotated in two sample files. In both files, the players have received different median scores by the annotators who assessed their dominance levels. The files were manually annotated in the ELAN annotation editor, ${ }^{3}$. Annotators marked in the time axis the extent of a speaker's gaze and its focus towards a specific direction. As soon as the gaze focus changed, a new segment was created. For each speaker, 3 values were employed: (a) gaze to speaker on the left, (b) gaze to speaker on the right, and (c) gaze away, i.e. in all the remaining cases where a speaker does not look at another co-speaker. A sample of an annotated file is presented in Figure 1. The analysis of the annotations focuses on (a) the number and average duration of gazes that a speaker gives and receives, (b) the number and average duration of mutual gazes between speakers and (c) a subset of the first case, the number and average duration of gazes to a participant who does not hold the floor. Also, while analysing the gaze behavior we make a distinction between the gaze of the facilitator and the gaze of the players, as we want to test whether different conversational roles entail different gaze patterns.

The annotation results suggest that the most dominant person receives more visual attention from the least dominant person than the other way round. Also, the least dominant person gazes more at the most dominant (when the latter isn't speaking) than the most dominant does when the least dominant isn't speaking. This could stem from the desire of the less dominant individual to be in tune with how their more dominant interlocutor is interpreting what is being said. Regarding the overlapping gaze between the moderator and each of the participants, the least dominant players are engaged in a larger amount of mutual gaze with the facilitator than the more dominant player did within the session.

It was additionally observed that the facilitator is more likely to gaze towards the least dominant players when they're silent rather than towards the most dominant ones when they are not speaking. This could be interpreted within the nature of the task: the facilitator's role is to help participants, if required, and also to encourage involvement in the task. A gaze towards the least dominant players may be a means of encouragement, an attempt to draw them into the interaction. This is further exemplified by the fact that the facilitator establishes more mutual gaze with the least dominant participant than with the most dominant participant. Contrary to findings in the literature about leadership, which claims that among a group of speakers with equal roles, the person who attracts the majority of

\footnotetext{
${ }^{3}$ https://tla.mpi.nl/tools/tla-tools/elan/ last accessed 10.05.2018.
}

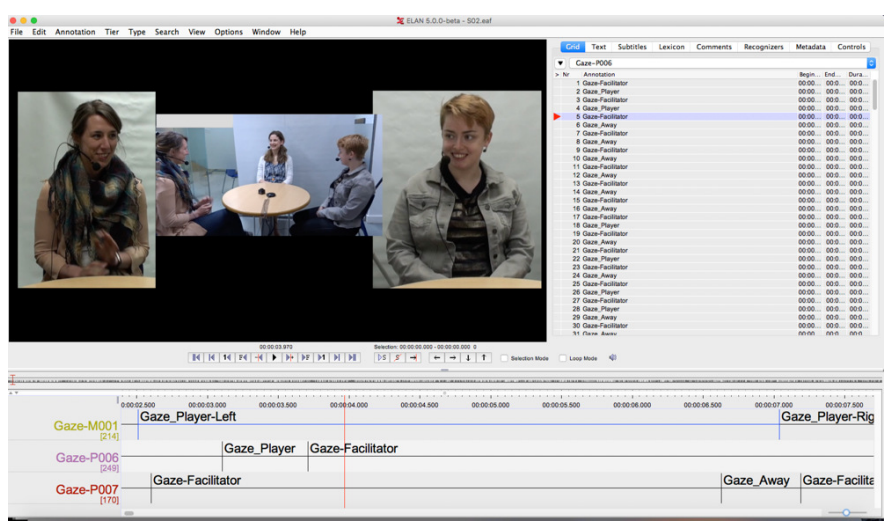

Fig. 1. Gaze annotation in ELAN

gazes is considered to have leadership assets, our observations suggest that a speaker of a certain role may be gazed mostly by a speaker of a different role, even if the former is not the most dominant.

Because the annotations were limited to 2 files in the corpus, we briefly presented our preliminary findings in this section, and we don't include gaze features in the models described in the next two sections. However, the findings drawn from the gaze annotations, while limited, provide interesting insights about the importance of the conversational role and the context that open up the potential for further investigation on the topic.

\section{INFLUENCES ON DOMINANCE}

\section{A. Dominance}

Using the locally-normed personality inventory scores described in $\S$ II-B and the aggregated (median) dominance rating of the five human annotators (see $\S$ II-C) we assessed the association beween the dominance rating (MEDIANDOMINANCE) and the personality traits. The dominance rating supplies a five-point ordinal scale, only four of which appear among the median values (Minimum $=1$, First Quartile $=2$, Median $=3$, Mean = 2.83, Third Quartile = 3.25, Maximum =4).

One might expect individuals with highest Extroversion scores to be among the most dominant and those with the highest Agreeableness scores to be least dominant. It is possible to construct arguments that Conscientiousness and Neuroticism should pattern with Extroversion and that Openness should pattern with Agreeableness.

Other qualities of linguistic interaction may also be imagined to have an impact on dominance scores - the highest number of words and highest number of words per minute are, by hypothesis, indicative of ownership of the floor (with fast speaking rate, it is more difficult for a dialogue partner to take a turn), and we imagine these correlate with high dominance; similarly, high values of turn duration indicate floor ownership, especially if coupled with a large number of turns owned.

\section{B. Personality traits and dominance}

The personality scale ranges from 0 to 100 . The data profile for each personality trait and for dominance is indicated in 
Table I, with histograms in Figure 2. Given that the personality scores are percentiles, derived versions binned these values, once using three break points $([0,25](25,50](50,75](75,100])^{4}$ and once using one break point ${ }^{5}([0,50](50,100])$.

TABLE I. MINIMUM, MAXIMUM, MEAN, AND QUARTILE VALUES FOR LOCALLY NORMED PERSONALITY SCORES OF PARTICIPANTS

\begin{tabular}{lrrrrrr} 
Trait & Min. & 1st Q. & Median & Mean & 3rd Q. & Max \\
\hline EXTROVERSION & 5.00 & 18.00 & 50.00 & 46.44 & 72.25 & 100.00 \\
AGREEABLENESS & 2.00 & 18.00 & 36.50 & 42.42 & 68.00 & 97.00 \\
CONSCIENTIOUSNESS & 2.00 & 24.00 & 50.00 & 47.00 & 72.25 & 90.00 \\
NEUROTICISM & 0.00 & 25.50 & 58.00 & 53.14 & 85.00 & 99.00 \\
OPENNESS & 0.00 & 22.00 & 50.00 & 50.47 & 84.00 & 96.00
\end{tabular}

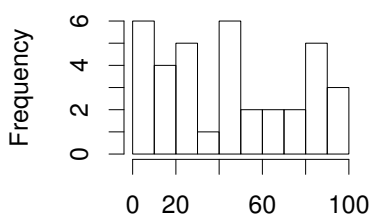

EXTROVERSION

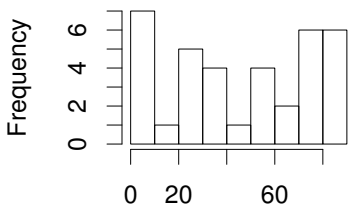

CONSCIENTIOUSNESS

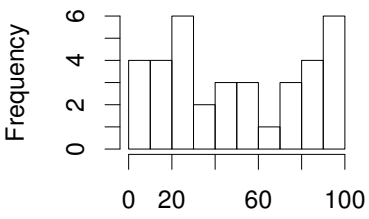

OPENNESS

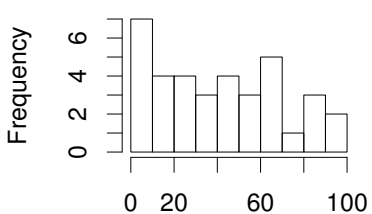

AGREEABLENESS

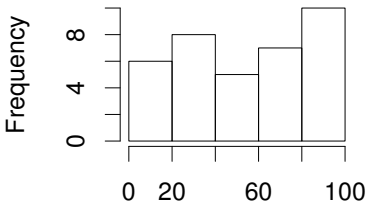

NEUROTICISM

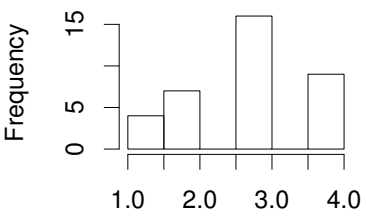

MedianDominance
Fig. 2. Histograms of Big-five trait scores depicting the distribution of scores (percentiles) in the data and Histogram of Median Dominance (bottom right) depicting the distribution of the 4 levels of Median Dominance

A model based on ordinal regression with Median Dominance as a dependent variable predicted by the unbinned five personality trait scores ${ }^{6}$ can be rejected as an adequate model $\left(p<2.02 * 10^{-7}\right)$. Stepped optimization from the initial model yields as a best fit one that retains only OPENNESS and

\footnotetext{
${ }^{4}$ These are named using initial capitals: Extroversion, Agreeableness, Conscientiousness, Neuroticism, Openness.

${ }^{5}$ These are named using entirely lowercase letters.

${ }^{6}$ Model. MedianDominanceGivenBIG5.polr <polr(factor(MedianDominance) EXTROVERSION+OPENNESS + CONSCIENTIOUSNESS+AGREEABLENESS+NEUROTICISM, HeSS=TRUE)
}

EXTROVERSION as factors (but still can be rejected as an adequate model $p<5.40 * 10^{-7}$ ). That these traits do not yield significance as predictors of Median Dominance can be seen in the plots that compare medians of the BIG5 traits at each level of Median Dominance provided in Fig. 3. Using the fourbinned versions of the personality trait variables (Fig. 4), one may see that the top quarter of Extroversion scores (left-hand plot) account for the highest values of Median Dominance and the top quarter of Agreeableness scores account for the lowest values of Median Dominance (and low values for Openness correspond to the lower values of Median Dominance). With two bins (right-hand plot of Fig. 4), only the extroversion and agreeableness effects remain visible.
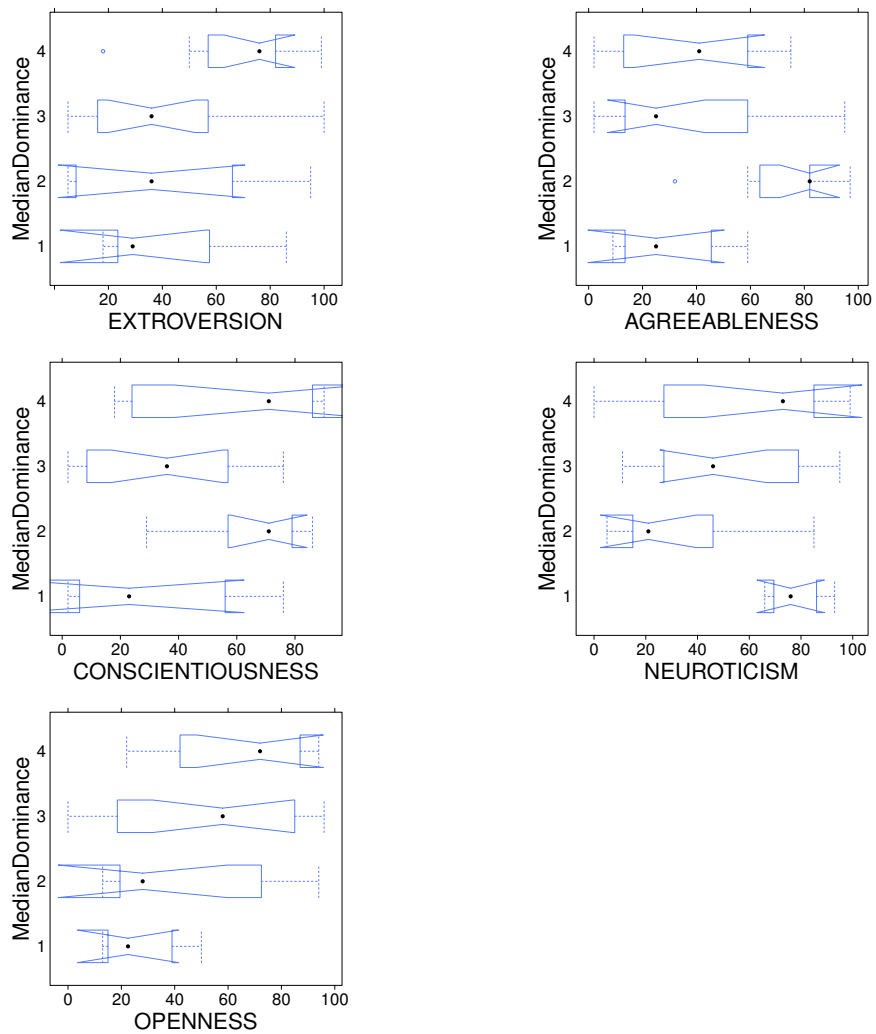

Fig. 3. Notched Box Plots depicting the independent effects of personality traits (percentiles) on the 4 levels of Median Dominance
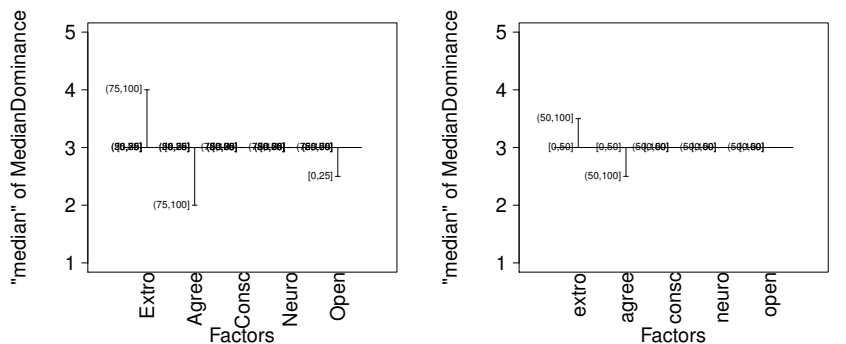

Fig. 4. Median Dominance given the BIG5 traits

In summary, only the main associations between highest extroversion and agreeableness and dominance (most, and 
least, respectively) are supported by the data, but without statistical significance.

\section{Conversational features and dominance}

We tested an ordinal regression model predicting Median Dominance on the basis of average turn duration, number of turns, number of words per minute and number of words as independent variables without interacting effects. ${ }^{7}$ Using stepped model optimization, the only remaining factor is number of words per minute; however, we can reject the null hypothesis that the model fits the data adequately $\left(p<2.4 * 10^{-5}\right)$. Nonetheless, within this model, number of words per minute is significant, with the interpretation that a one unit increase in number of words per minute increases the odds of having a higher dominance rating are 1.11 times larger than having a lower dominance rating. Box and whisker plots of the individual effects on median dominance are provided in Fig. 5; these indicate substantial overlap in the inner quartiles around the median for each level of median dominance, in each case except number of words per minute.
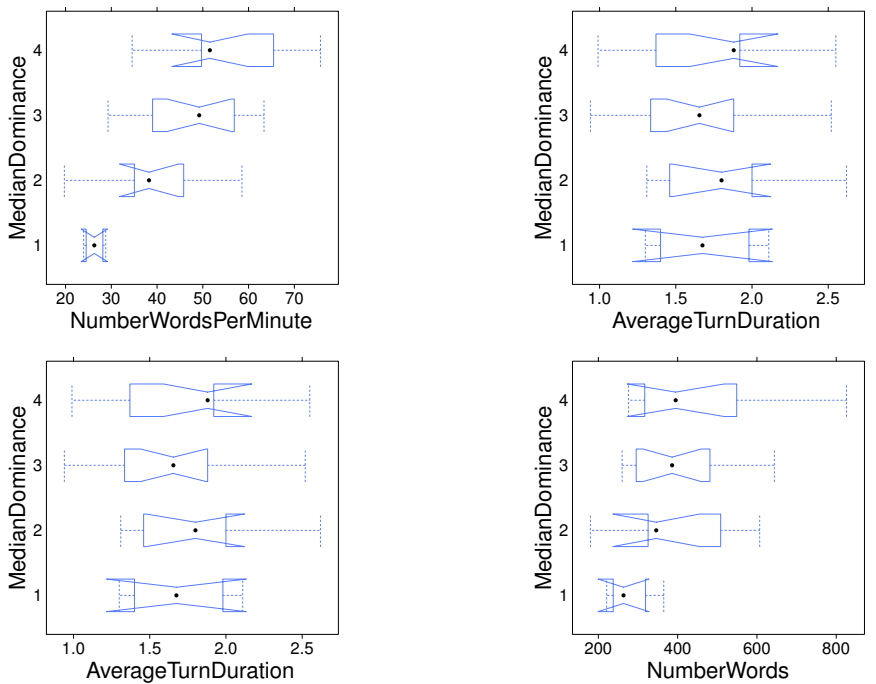

Fig. 5. Notched Box Plots depicting independent effects of conversational factors on the 4 levels of Median Dominance

An ordinal regression model that predicts median dominance on the basis of words per minute and each of extroversion, opennness and agreeableness can be rejected as an adequate model. Stepped model optimization settles on a model that retains the interaction between number of words per minute and extroversion and the interaction between number of words per minute and agreeableness; however, the only coefficient with significance $(p<0.001)$ is number of words per minute. We conclude that these interaction models do not have explanatory value in this data.

Thus, in this data set, the personality traits of extroversion and openness on one hand and agreeableness on the other hand appear to have opposed influences on dominance (the former increase together with dominance, but the as the latter

\footnotetext{
${ }^{7}$ polr(factor(MedianDominance) AverageTurnDuration+ Number. of . Turns +NumberWordsPerMinute+NumberWords, Hess $=\mathrm{TRUE}$ )
}

increases, dominance decreases). Additionally, increases in the number of words per minute predict increase in median dominance. It must be emphasized that this measure is relativized to the total conversation duration, and thus relates to the amount of floor time that the speaker has, rather than whether they speak quickly or not.

\section{INFLUENCES OF DOMINANCE}

As constructed in this work, dominance is a response variable. Nonetheless, it is interesting to assess the extent to which dominance is adequate predictor of other quantities. In the first instance, it is helpful to explore these relationships using the inverse of the plot of Fig. 4, as provided in Fig.6. The only case where ordered levels of median dominance is systematic in relation to personality trait variables is openness, although the extroversion effect involves a very nearly ordered relation on levels of median dominance.
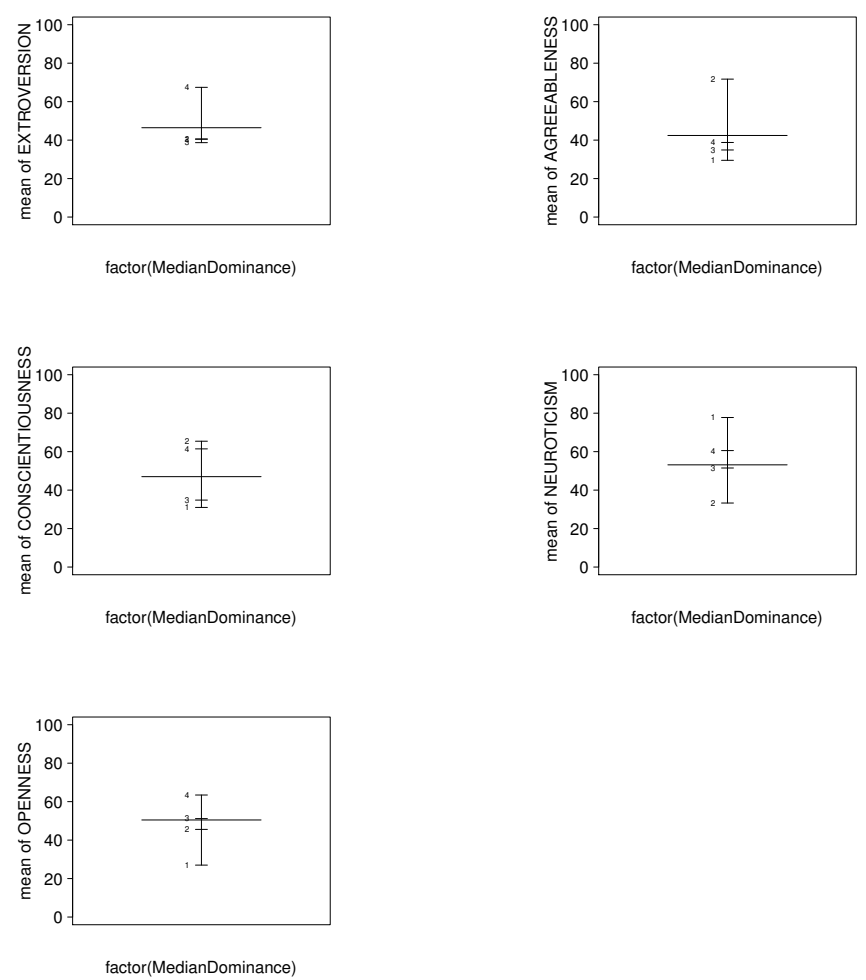

Fig. 6. BIG5 traits (percentiles) given Median Dominance

The data distributions shown in Fig.2 suggest that regression techniques are not suitable to the data. However, applying the Kruskal-Wallis rank-test of means across categories (that is, thinking of median dominance as a categorical variable with three levels, without regard to order), significance obtains for conscientiousness $\left(\chi^{2}=8.216, d f=3, p<0.05\right)$, and agreeableness $\left(\chi^{2}=9.203, d f=3, p<0.05\right)$. Nonetheless, given the construction of median dominance, it is difficult to overlook order in its construction. A conservative generalization that follows is that being below the neutral point in dominance, but above the minimum level of dominance predicts being high in agreeableness and well the middle 
percentiles in conscientiousness. A stronger effect that respects ordering of levels of median dominance is upon number of words per minute $\left(\chi^{2}=13.59, d f=3, p<0.005\right)$. Effects on average turn duration, number of words, and number of turns are not significant.

\section{Discussion}

We have explored bi-directional relations between median dominance scores and both personality traits and conversational profile factors. The fact that the models predicting median dominance are not adequate to the data suggests that other variables than we discussed in $\S$ III have an important role in explaining the distribution of median dominance scores. ${ }^{8}$ Nonetheless, high extroversion predicts high dominance scores and so does high openness, to a lesser extent; high agreeableness scores predict low dominance scores. Contributing a large number of words per minute of overall dialogue predicts high dominance. In the other direction, high dominance predicts high openness and a greater number of words per minute of the dialogue.

We have also conducted a small-scale study associating gaze patterns to dominance; preliminary results indicate that the conversational role of the speakers is important in the interpretation of the gaze focus on the most or least dominant participant.

The psychological perspective (conversational dominance, personality, cognitive capabilities, etc.) of multimodal interaction participants directly influences the nature of infocommunication as well as the the kind of information that is transmitted [23]. In this respect, we believe that the quantification of dominance forms an inherent part of the research in Linguistic and Behavioural Interaction Analysis, a topic of Cognitive Infocommunications, specifically related to the intra-cognitive communication mode and the sensor-sharing communication type identified in the field of CogInfoCom [24].

\section{ACKNOWLEDGMENT}

This research is supported by the European Union's Horizon 2020 research and innovation programme under the Marie Sklodowska-Curie grant agreement No 701621 (MULTISIMO) and by Science Foundation Ireland (SFI) through the CNGL Programme (Grant 12/CE/I2267 and 13/RC/2106) in the ADAPT Centre (www.adaptcentre.ie).

\section{REFERENCES}

[1] H. Itakura, "Describing conversational dominance," Journal of Pragmatics, vol. 33, pp. 1859-1880, 2001

[2] D. Jayagopi, H. Hung, C. Yeo, and D. Gatica-Perez, "Modeling dominance in group conversations from non-verbal activity cues," IEEE Transactions on Audio, Speech and Language Processing, vol. 17, no. 3, pp. 501-513, 2009.

[3] N. E. Dunbar and J. K. Burgoon, Measuring Nonverbal Dominance Lawrence Erlbaum Associates, Publishers, 2005, p. pages.

[4] - "Perceptions of power and interactional dominance in interpersonal relationships," Journal of Social Personal Relationships, vol. 22 no. 2, pp. 207-233, 2005.

\footnotetext{
${ }^{8}$ Among these, could be the aggregation of median dominance from the five annotators' ratings.
}

[5] D. Sanchez-Cortes, O. Aran, M. S. Mast, and D. Gatica-Perez, "A nonverbal behavior approach to identify emergent leaders in small groups," IEEE Transactions on Multimedia, vol. 14, no. 3-2, pp. 816832, Jun. 2012.

[6] Y. Nakano and Y. Fukuhara, "Estimating conversational dominance in multiparty interaction," in Proceedings of the 14th ACM International Conference on Multimodal Interaction, ser. ICMI '12. New York, NY, USA: ACM, 2012, pp. 77-84. [Online]. Available: http://doi.acm.org/10.1145/2388676.2388699

[7] M. S. Mast, "Dominance as expressed and inferred through speaking time: A meta-analysis," Human Communication Research, vol. 28, no. 3, pp. 420-450, 2002. [Online]. Available: http: //dx.doi.org/10.1111/j.1468-2958.2002.tb00814.x

[8] A. Kendon, "Some functions of gaze-direction in social interaction," Acta Psychologica, vol. 26, pp. 22 - 63, 1967. [Online]. Available: http://www.sciencedirect.com/science/article/pii/0001691867900054

[9] R. Rienks and D. Heylen, "Dominance detection in meetings using easily obtainable features," in Machine Learning for Multimodal Interaction, S. Renals and S. Bengio, Eds. Berlin, Heidelberg: Springer Berlin Heidelberg, 2006, pp. 76-86.

[10] N. E. Dunbar, A. R. Jr., and J. K. Burgoon, "The effects of participation on the ability to judge deceit," Communication Reports, vol. 16, no. 1, pp. 23-33, 2003

[11] A. P. Kalma, L. Visser, and A. Peeters, "Sociable and aggressive dominance: Personality differences in leadership style?" The Leadership Quarterly, vol. 4, no. 1, pp. 45 - 64, 1993. [Online]. Available: http://www.sciencedirect.com/science/article/pii/104898439390003C

[12] H. Hassan, S. Asad, and Y. Hoshino, "Determinants of leadership style in big five personality dimensions," Universak Journal of Management, vol. 4, no. 4, pp. 161-179, apr 2016.

[13] L. Laustsen and M. B. Petersen, "Perceived conflict and leader dominance: Individual and contextual factors behind preferences for dominant leaders," Political Psychology, vol. 38, no. 6, pp. 1083-1101, 2017. [Online]. Available: https://onlinelibrary.wiley.com/doi/abs/10. 1111/pops. 12403

[14] B. Ekehammar, N. Akrami, M. Gylje, and I. Zakrisson, "What matters most to prejudice: Big five personality, social dominance orientation, or right-wing authoritarianism?" European Journal of Personality, vol. 18, pp. 463-482, 2004.

[15] M. Koutsombogera and C. Vogel, "Modeling collaborative multimodal behavior in group dialogues: The MULTISIMO corpus," in Proceedings of the Eleventh International Conference on Language Resources and Evaluation (LREC 2018). Paris, France: European Language Resources Association (ELRA), 2018, pp. 2945-2951.

[16] M. C. Ashton, K. Lee, and S. V. Paunonen, "What is the central feature of extraversion? social attention versus reward sensitivity," Journal of personality and social psychology, vol. 83, no. 1, pp. 245-252, 2002.

[17] A. Mehrabian, "Analysis of the big five personality factors in terms of the pad temperament model," Australian Journal of Psychology, vol. 48, no. 2, pp. 86-92, 1996. [Online]. Available: https://onlinelibrary.wiley.com/doi/abs/10.1080/00049539608259510

[18] C. G. Sibley and J. Duckitt, "Personality and prejudice: A metaanalysis and theoretical review," Personality and Social Psychology Review, vol. 12, no. 3, pp. 248-279, 2008, pMID: 18641385. [Online]. Available: https://doi.org/10.1177/1088868308319226

[19] D. C. Richardson and R. Dale, "Looking to understand: the coupling between speakers' and listeners' eye movements and its relationship to discourse comprehension," Cognitive Science, vol. 29, pp. 39-54, 2005.

[20] L. R. Goldberg, "The development of markers for the big-five factor structure," Psychological Assessment, vol. 4, no. 1, pp. 26-42, 1992.

[21] O. P. John, E. M. Donahue, and R. L. Kentle, "The big five inventory versions 4a and 54," 1991.

[22] O. P. John, L. P. Naumann, and C. J. Soto, "Paradigm shift to the integrative big five trait taxonomy," Handbook of personality: Theory and research, vol. 3, pp. 114-158, 2008.

[23] G. Sallai, "Defining infocommunications and related terms," Acta Polytechnica Hungarica, vol. 9, no. 6, pp. 5-15, 2012.

[24] P. Baranyi and A. Csapo, "Definition and synergies of cognitive infocommunications," Acta Polytechnica Hungarica, vol. 9, no. 1, pp. $67-83,2012$ 